\title{
Low Awareness of Nonalcoholic Fatty Liver Disease in a Population-Based Cohort Sample: the CARDIA Study
}

\author{
Erin R. Cleveland, $M D^{7}$, Hongyan Ning, $M D, M S^{2}$, Miriam B. Vos, $M D^{3}$, \\ Cora E. Lewis, MD, MSPH, FACP, FAHA ${ }^{4}$, Mary E. Rinella, $M D^{7}$, John Jeffrey Carr, $M D^{5}$, \\ Donald M. Lloyd-Jones, MD, SCM, FACC, FAHA ${ }^{2,6}$, and Lisa B. VanWagner, MD, MSC, FAST, FAHA ${ }^{1,2}$
}

\footnotetext{
'Department of Medicine-Division of Gastroenterology \& Hepatology, Northwestern University Feinberg School of Medicine, Chicago, IL, USA; ${ }^{2}$ Department of Preventive Medicine, Northwestern University Feinberg School of Medicine, Chicago, IL, USA; ${ }^{3}$ Department of Pediatrics-Division of Gastroenterology, Hepatology \& Nutrition, Emory University, Atlanta, GA, USA; ${ }^{2}$ Department of Medicine-Division of Preventive Medicine, University of Alabama Birmingham, Birmingham, AL, USA; ${ }^{5}$ Department of Radiology, Vanderbilt University, Nashville, TN, USA; ${ }^{6}$ Department of Medicine-Division of Cardiology, Northwestern University Feinberg School of Medicine, Chicago, IL, USA.
}

BACKGROUND: Nonalcoholic fatty liver disease (NAFLD) is the most common chronic liver disease in the United States, yet little is known about NAFLD awareness in individuals with incidental fatty liver on imaging.

OBJECTIVE: To assess the level of awareness of imagingdefined NAFLD among individuals with and without metabolic risk factors.

DESIGN: Cross-sectional analysis within a prospective longitudinal population-based cohort study conducted in four U.S. cities.

PARTICIPANTS: Adults age 43 to 55 years enrolled in the Coronary Artery Risk Development in Young Adults (CARDIA) Study who underwent computed tomography and a personal health questionnaire at the year 25 exam (2010-2011, $n=2788)$.

MAIN MEASURES: NAFLD was defined as liver attenuation $\leq 51$ Hounsfield units after exclusion of other causes of liver fat. Participants were considered "NAFLD aware" if they reported being told previously by a doctor or nurse that they had "fatty liver."

KEY RESULTS: NAFLD prevalence was 23.9\%. Only 16 of 667 (2.4\%) participants with CT-defined NAFLD were aware of a NAFLD diagnosis. NAFLD aware participants were more likely to be white $(81.3 \%$ vs. $53.5 \%, p=0.03)$ and have the metabolic syndrome $(87.5 \%$ vs. $59.3 \%, p=$ 0.02 ) and/or hypertension ( $75.0 \%$ vs. $50.2 \%, p=0.05)$. In multivariable analyses adjusted for demographics, metabolic syndrome and hypertension remained predictive of NAFLD awareness.

CONCLUSION: There is low awareness of NAFLD among individuals with hepatic steatosis on imaging, even among those with metabolic risk factors. These findings highlight an opportunity to raise public and practitioner awareness of NAFLD with the goal of increasing diagnosis and implementing early treatment strategies.

Electronic supplementary material The online version of this article (https://doi.org/10.1007/s11606-019-05340-9) contains supplementary material, which is available to authorized users.

Received February 5, 2019

Revised July 11, 2019

Accepted August 21, 2019

Published online October 8, 2019
KEY WORDS: NAFLD; computed tomography; NASH; metabolic syndrome; hepatic steatosis.

$\mathrm{J}$ Gen Intern Med 34(12):2772-8

DOI: $10.1007 / \mathrm{s} 11606-019-05340-9$

(c) Society of General Internal Medicine 2019

\section{INTRODUCTION}

Nonalcoholic fatty liver disease (NAFLD) is a growing health burden in the United States (U.S.) and internationally with the global prevalence estimated at $25.2 \%{ }^{1}$ It is the most common cause of chronic liver disease in the U.S., accounting for up to $75 \%$ of all liver disease. ${ }^{2}$ NAFLD occurs more frequently among patients with the metabolic syndrome and as the prevalence of obesity, diabetes mellitus, and hypertension increases, the NAFLD burden continues to rise in parallel. ${ }^{3}$

Patients with NAFLD have higher overall mortality compared with matched control populations without NAFLD. ${ }^{4}$ Cardiovascular disease is the most common cause of death in NAFLD independent of other metabolic comorbidities. ${ }^{3}$ NAFLD represents a disease spectrum ranging from isolated hepatic steatosis, which carries relatively lower risk of liver disease progression, to steatosis plus inflammation (nonalcoholic steatohepatitis, NASH) with or without fibrosis, which may eventually progress to cirrhosis. ${ }^{3}$ Patients with histologically confirmed NASH are at highest risk of liver-related morbidity and mortality. ${ }^{5}$

NAFLD is diagnosed by the detection of fatty liver on imaging after exclusion of other causes of liver fat, ${ }^{3}$ and thus is commonly an incidental finding on imaging studies obtained for other indications. Little is known about NAFLD awareness in nonclinical populations with fatty liver on imaging. Despite numerous cross-sectional population-based studies that estimate the prevalence of NAFLD in the U.S. (e.g., National Health and Nutrition Examination Survey, Multiethnic Study of Atherosclerosis, Framingham), none have published data describing participant awareness of an imagingbased NAFLD diagnosis at the time of NAFLD assessment. 
Here we aimed to assess the prevalence of participant awareness of imaging-defined NAFLD among individuals with and without metabolic risk factors in a biracial, population-based, well-phenotyped prospective longitudinal cohort study. Secondary objectives included examining the effect of metabolic risk factors, race, socioeconomic status, and sex on the prevalence of NAFLD awareness.

\section{METHODS}

\section{Study Design and Sample}

The Coronary Artery Risk Development in Young Adults (CARDIA) Study is a multicenter, prospective, longitudinal cohort study of lifestyle and cardiovascular risk factors in young adults. Between 1985 and 1986, 5115 participants were recruited across four U.S. cities (Birmingham, AL; Chicago, IL; Minneapolis, MN; Oakland, CA). The study design has been previously published. ${ }^{6}$ Participants were balanced by sex, race (white or black), age (18-24 years old or 2530 years old), and education level ( $\leq$ high school or $>$ high school). Follow-up visits were conducted at years 2, 5, 7, 10, 15 , and 25 with retention of $72 \%$ of participants at year 25 (2010-2011). The CARDIA Study is ongoing (year 30 exam completed in 2016). Informed consent was obtained at each follow-up examination.

The present report includes 3498 CARDIA participants (age 43-55) who participated in the year 25 exam between June 2010 and August 2011 and underwent cross-sectional imaging with noncontrast computed tomography (CT) scanning of the abdomen. Participants were excluded if they were pregnant, weighed greater than 450 pounds or were unable to fit in the CT scanner, or had missing covariates $(n=339)$. Participants with other self-reported causes of liver fat, including heavy alcohol use (defined as $>14$ standard drinks per week for women or $>21$ standard drinks per week for men), human immunodeficiency virus (HIV), hepatitis $\mathrm{C}$ virus (HCV), and medications known to cause hepatic steatosis (e.g., amiodarone, diltiazem, methotrexate, valproate, tamoxifen), were also excluded ( $n=434)$ (Fig. 1).

\section{Data Collection}

Standardized protocols for data collection were consistent across all study centers and have been previously described. ${ }^{7}$ The CT scans were performed using multidetector CT scanners from either General Electric (GE 750HD 64 and GE LightSpeed VCT 64 Birmingham and Oakland Centers, respectively; GE Healthcare, Waukesha, WI) or Siemens (Sensation 64, Chicago and Minneapolis Centers; Siemens Medical Solutions, Erlangen, Germany). Liver attenuation was measured in three regions of the right lobe of the liver across three separate CT slices and was reported as the average of nine measurements. Any hepatic steatosis was defined as liver attenuation $\leq 51$ Hounsfield units, which approximates a liver/ spleen ratio $<1.0 .^{8}$ Moderate-to-severe hepatic steatosis was defined as liver attenuation $\leq 40$ Hounsfield units. ${ }^{9}{ }^{10}$ Image analysis and quality control were performed at a core reading center (Wake Forest University Health Sciences, WinstonSalem, NC). Quality control reviews were conducted on approximately $5 \%$ of studies. The interclass correlation coefficient (ICC) between different readers on this randomly selected sample of 156 participants was 0.975 for liver attenuation, indicating high reproducibility of CT-measured liver attenuation in this study.

Demographic and medical characteristics of the participants were obtained from the CARDIA database. Variables collected included age, sex, income, education level, body mass index (BMI), blood pressure, medications, daily alcohol use, level of physical activity, medical comorbidities, and variables related to access to healthcare (e.g., identified usual source of medical care and perceived difficulty obtaining health services).

Body weight was measured to the nearest $0.2 \mathrm{~kg}$ with a calibrated balance beam scale. Height was measured with a vertical ruler to the nearest $0.5 \mathrm{~cm}$. Seated blood pressure was measured three times at 1-min intervals after $5 \mathrm{~min}$ of resting, and the second and third measurements were averaged. Fasting blood was drawn in the seated position, separated, and plasma frozen to $70{ }^{\circ} \mathrm{C}$ prior to analysis in a central laboratory. ${ }^{6}$ Glucose was assayed using the hexokinase method and insulin by the Elecsys sandwich immunoassay. Total cholesterol, high-density lipoprotein (HDL) cholesterol, and triglycerides were measured enzymatically at the Northwest Lipid Laboratory. ${ }^{11}$ LDL cholesterol was calculated using the Friedewald equation.

Obesity was defined as body mass index (BMI) $\geq 30 \mathrm{~kg} /$ $\mathrm{m}^{2} .{ }^{12}$ Hypercholesterolemia was defined as a total cholesterol level of $\geq 240 \mathrm{mg} / \mathrm{dl}$. Hypertension was defined as antihypertensive medication use and/or systolic blood pressure $\geq$ $140 \mathrm{mmHg}$ or diastolic blood pressure $\geq 90 \mathrm{mmHg}$. Diabetes was defined as fasting plasma glucose $\geq 126 \mathrm{mg} / \mathrm{dl}$, treatment with insulin or hypoglycemic agent, 2-h post-challenge glucose $\geq 200 \mathrm{mg} / \mathrm{dl}$, and/or $\mathrm{HbA}_{1 \mathrm{c}} \geq 6.5 \%$. The modified National Cholesterol Education Program Adult Treatment Panel III criteria were used to define the metabolic syndrome. ${ }^{13}$ To quantify physical activity (reported as exercise units (EU)), the CARDIA physical activity history questionnaire was used, which was an interviewer-based self-report of duration and intensity of participation in 13 categories of exercise over the previous 12 months. ${ }^{14}$ As a reference, $300 \mathrm{EU}$ approximates $150 \mathrm{~min}$ of moderate-intensity activity per week or $30 \mathrm{~min}$ of moderate-intensity activity 5 days per week. ${ }^{14}$

\section{Survey Design}

Surveys were administered prior to CT imaging to all study participants during the year 25 exam to determine whether they were aware of any prior diagnosis of NAFLD. Specifically, they were asked to respond to the question "Has a doctor 


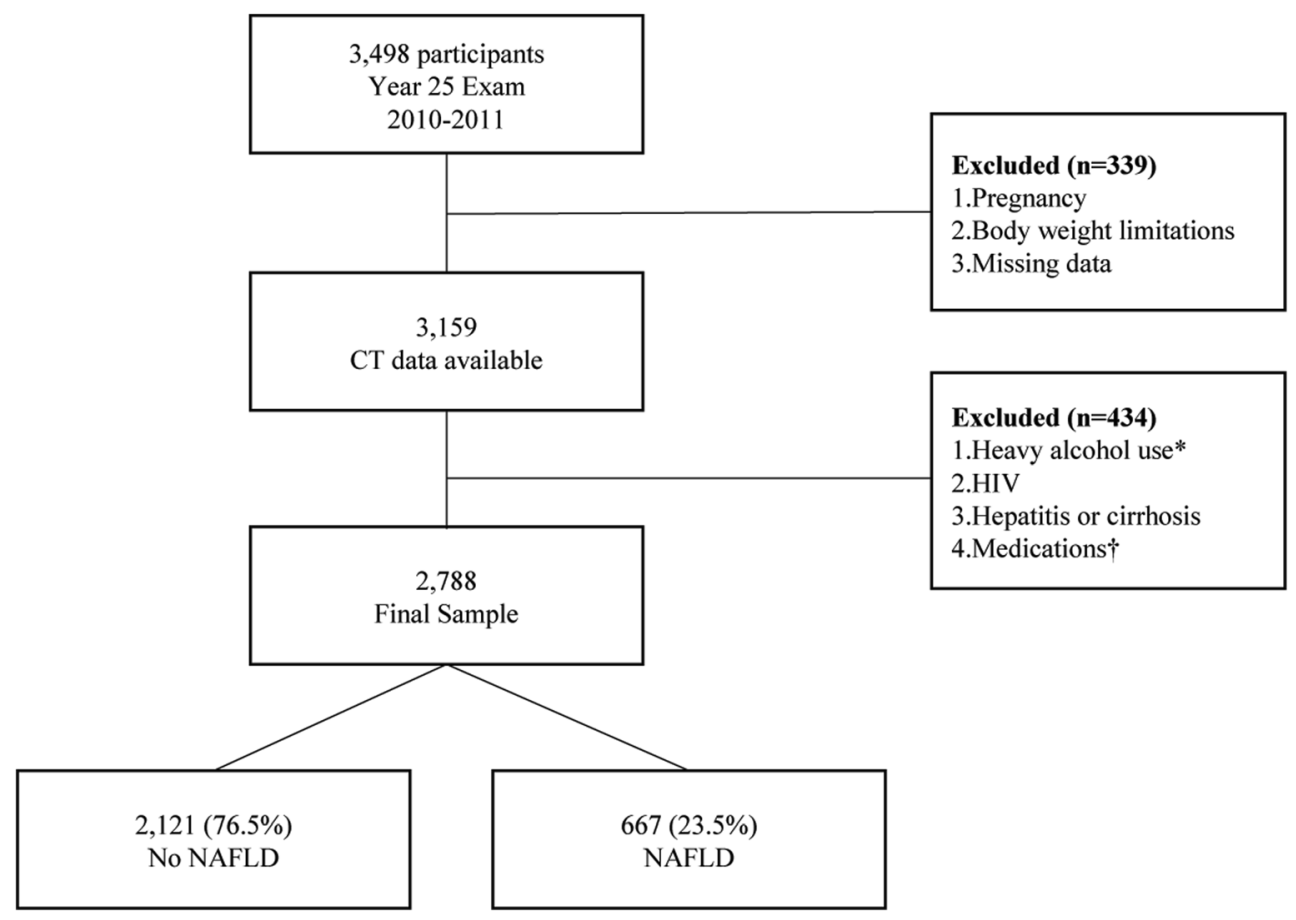

Figure 1 Study design. NAFLD was defined as mean liver attenuation $\leq \mathbf{5 1}$ Hounsfield units (equivalent to a liver spleen ratio $<1.0$ ) after exclusion of other causes of fatty liver. CT, computed tomography; NAFLD, nonalcoholic fatty liver disease; HIV, human immunodeficiency virus. Asterisk symbol denotes heavy alcohol use defined as $>14$ drinks/week for women, $>\mathbf{2 1}$ drinks/week for men. Dagger symbol denotes medications amiodarone, diltiazem, methotrexate, valproate, tamoxifen

or nurse ever said that you have liver disease?" For all "yes" responses, participants were then asked to specify, "What type of liver disease: (a) hepatitis (type A, B, C or unsure), (b) cirrhosis, (c) fatty liver, (d) other or (e) don't know." Those participants who responded "yes" to the first question and specified "fatty liver" in the second question were considered to be "NAFLD aware." All other participants were "NAFLD unaware" (Fig. 1).

\section{Statistical Analysis}

Characteristics were compared by self-reported NAFLD status (unaware vs. aware) using generalized linear models for continuous variables and chi-square or Fisher's exact test for categorical variables. Logistic regression models were used to quantify cross-sectional associations between metabolic covariates and self-reported NAFLD status (aware vs. unaware). Covariates in the multivariable model were chosen a priori for clinical importance. The multivariable model was adjusted for age, race, sex, education, and income level. Interaction terms were generated between race and sex in terms of NAFLD awareness status. Because no interactions by race or sex were noted, all models include only main effects for race and sex. Sensitivity analysis was performed a priori that included all participants who reported any history of known liver disease regardless of subtype as well as participants with moderate-to-severe hepatic steatosis. A $p$ value $<0.05$ was considered statistically significant. Analyses were performed using SAS 9.2 (SAS institute, Cary, NC).

\section{IRB Approval}

Institutional Review Board (IRB) approval was obtained from all study sites prior to data collection. All CARDIA documents are publicly available at: https://www.cardia.dopm.uab.edu/.

\section{RESULTS}

Among 2788 participants who were included in this study, 667 (23.9\%) had any CT-defined NAFLD and 272 (9.8\%) had moderate-to-severe NAFLD. Among those with any NAFLD, the mean participant age was 50.4 (SD, 3.6 years), 301 (45.1\%) were female, and 306 (45.9\%) were black. The vast majority of participants reported having a usual source of medical care $(92.6 \%)$. In the NAFLD group, $50.8 \%$ had a diagnosis of hypertension, $32.6 \%$ had hyperlipidemia, and $29.2 \%$ had diabetes. The mean body mass index (BMI) was $35.2\left(\mathrm{SD}, 7.3 \mathrm{~kg} / \mathrm{m}^{2}\right)$ with $76.5 \%$ of participants being classified as obese (BMI $\geq 30)$. Four hundred $(60.0 \%)$ met criteria for the metabolic syndrome (Appendix Table 1 online).

Although the prevalence of any NAFLD in CARDIA was $23.9 \%$, only 16 of 667 participants $(2.4 \%)$ with any CTdefined NAFLD were aware of a NAFLD diagnosis. Even when the definition was broadened to include any selfreported liver disease, only 34 participants $(5.1 \%)$ reported knowing about a prior diagnosis. Among patients with moderate-severe hepatic steatosis, 5 of $272(1.8 \%)$ participants were aware of a NAFLD diagnosis. 
Table 1 Demographic and Clinical Characteristics of "NAFLD Aware" and "NAFLD Unaware" Participants

\begin{tabular}{|c|c|c|c|c|}
\hline Characteristics & $\begin{array}{l}\text { Participants with any NAFLD } \\
(N=667)^{*}\end{array}$ & $\begin{array}{l}\text { NAFLD aware } \\
(N=16)^{*}\end{array}$ & $\begin{array}{l}\text { NAFLD unaware } \\
(N=651)^{*}\end{array}$ & $p$ value $^{\dagger}$ \\
\hline Age, years, mean (SD) & $50.4(3.6)$ & $50.8(4.2)$ & $50.4(3.6)$ & 0.70 \\
\hline Women $(\%)$ & 45.1 & 62.5 & 44.7 & 0.16 \\
\hline White $(\%)$ & 54.1 & 81.3 & 53.5 & 0.03 \\
\hline \multicolumn{5}{|l|}{ Socioeconomic status } \\
\hline Highest grade completed, mean (SD) & $14.9(2.6)$ & $15.8(2.6)$ & $14.8(2.6)$ & 0.17 \\
\hline Income $>\$ 50,000(\%)$ & 60.1 & 75.0 & 59.8 & 0.22 \\
\hline \multicolumn{5}{|l|}{ Access to medical care } \\
\hline Report regular source of medical care $(\%)$ & 92.6 & 100.0 & 92.5 & 0.25 \\
\hline Report difficulty accessing healthcare $(\%)$ & 11.7 & 6.3 & 11.8 & 0.88 \\
\hline $\mathrm{BMI}\left(\mathrm{kg} / \mathrm{m}^{2}\right)$, mean (SD) & $35.2(7.3)$ & $34.9(6.8)$ & $35.2(7.3)$ & $\begin{array}{l}0.00 \\
0.88\end{array}$ \\
\hline Obese, $\mathrm{BMI}>30(\%)$ & 76.5 & 75.0 & 76.6 & 0.88 \\
\hline Waist circumference $(\mathrm{cm})$, mean $(\mathrm{SD})$ & $108.5(14.8)$ & $107.3(14.3)$ & $108.5(14.8)$ & 0.73 \\
\hline Glomerular filtration rate $\left(\mathrm{ml} / \mathrm{min} / 1.73 \mathrm{~m}^{2}\right)$, mean $(\mathrm{SD})$ & $97.6(22.8)$ & $95.8(20.3)$ & $97.6(22.9)$ & 0.75 \\
\hline \multicolumn{5}{|l|}{ Comorbidities (\%) } \\
\hline Hypercholesterolemia ${ }^{\S}$ & 32.6 & 18.8 & 32.9 & 0.23 \\
\hline Hypertension" & 50.8 & 75.0 & 50.2 & 0.05 \\
\hline Diabetes mellitus ${ }^{\text {II }}$ & 29.2 & 25.0 & 29.3 & 0.71 \\
\hline Metabolic syndrome** & 60.0 & 87.5 & 59.3 & 0.02 \\
\hline \multicolumn{5}{|l|}{ Medication usage (\%) } \\
\hline Diabetes medication & 19.1 & 12.5 & 19.3 & 0.50 \\
\hline Hypertension medication & 41.1 & 68.8 & 40.4 & 0.02 \\
\hline Lipid-lowering medication & 24.1 & 18.8 & 24.3 & 0.61 \\
\hline Alcohol use $(\%)$ & 46.5 & 35.7 & 46.8 & 0.41 \\
\hline Daily alcohol use, g/day, mean (SD) & $5.0(7.5)$ & $4.0(6.8)$ & $5.0(7.5)$ & 0.62 \\
\hline Physical activity (exercise units/week), mean (SD) & $294(256)$ & $232(164)$ & $296(257)$ & 0.32 \\
\hline
\end{tabular}

Any NAFLD was defined as mean liver attenuation $\leq 51$ Hounsfield units (equivalent to a liver spleen ratio < 1.0) after exclusion of other causes of fatty liver

*N as listed except where data are missing

"p value denotes differences between "NAFLD aware" and "NAFLD unaware"

"Responded "hard" or "very hard" in response to survey question, "How hard is it to get needed health services?"

${ }^{\S}$ Total cholesterol $\geq 240 \mathrm{mg} / \mathrm{dl}$

"Antihypertensive medication use and/or systolic blood pressure $\geq 140 \mathrm{mmHg}$ or diastolic blood pressure $\geq 90 \mathrm{mmHg}$

${ }^{\uparrow}$ Fasting plasma glucose $\geq 126 \mathrm{mg} / \mathrm{dl}$, treatment with insulin or hypoglycemic agent, 2-h post-challenge glucose $\geq 200 \mathrm{mg} / \mathrm{dl}$, and/or hemoglobin $\mathrm{A}_{1 \mathrm{c}} \geq$ $6.5 \%$

**Defined using Adult Treatment Panel III criteria

Participants who were NAFLD aware were more likely to be white than participants who were unaware of a NAFLD diagnosis $(81.3 \%$ vs. $54.1 \%, p=0.028)$. There were no significant differences in age, sex, education level, income, or reported access to medical care between the NAFLD aware and NAFLD unaware groups. There were also no differences in average alcohol use or amount of physical activity between the groups (Table 1).

Participants who were aware of a NAFLD diagnosis were more likely to have the metabolic syndrome than NAFLD unaware participants $(87.5 \%$ vs. $59.3 \%, p=$ $0.023)$. NAFLD aware participants were also more likely to have hypertension $(75.0 \%$ vs. $50.2 \%, p=0.050)$ than unaware participants. Individually, there were no significant differences in BMI, waist circumference, prevalence of diabetes, or prevalence of hyperlipidemia between the NAFLD aware and unaware groups.

In multivariable analyses adjusted for demographics (age, race, sex, education, and income), the presence of the metabolic syndrome and hypertension was associated with higher NAFLD awareness (OR 5.39, 95\% CI 1.2-24.2 and OR 4.99, 95\% CI 1.52-16.37, respectively). Despite this association, the prevalence of NAFLD awareness even among participants with the metabolic syndrome was low (3.5\%). In sensitivity analyses using self-report of any liver disease $(n=34)$, these associations did not change (data not shown).

\section{DISCUSSION}

Our data demonstrate strikingly low awareness of fatty liver among a population-based sample of asymptomatic, middle-age adults with hepatic steatosis on imaging. The presence of the metabolic syndrome and hypertension was associated with increased NAFLD awareness; however, low awareness was present even among participants with metabolic risk factors that are associated with increased risk for severe liver disease and death. In conjunction with the high prevalence of hepatic steatosis noted among this sample of U.S. adults, the lack of disease awareness highlights the need for improved education and allocation of resources in our approach to the growing NAFLD epidemic.

Study participants' lack of awareness of a NAFLD diagnosis is likely multifactorial. Possible factors include the asymptomatic nature of NAFLD early in the disease course, the lack of current screening guidelines for the disease, and poor understanding of NAFLD among patients and providers. Be- 
cause hepatic steatosis generally does not lead to symptoms until the disease has progressed to the point of advanced fibrosis or cirrhosis, the disease may go undetected for years. Even among those following regularly with a physician, NAFLD is often detected incidentally on imaging exams done for other purposes.

In this study, hepatic steatosis was an incidental finding rather than a diagnosis made as part of the workup of a clinical abnormality, such as abnormal liver chemistries. It is important to note that elevated liver chemistries are not an appropriate diagnostic tool for NAFLD as many patients with NAFLD will have normal liver chemistries, and elevated liver chemistries have poor prognostic ability for the diagnosis of NASH. ${ }^{3}$,

${ }^{15}$ In contrast, liver ultrasound or transient elastography is more sensitive than liver chemistries for detection of hepatic steatosis; however, their effectiveness as screening tools has not been systematically investigated. ${ }^{15}$

Importantly, current guidance from the American Association for the Study of Liver Diseases (AASLD) does not recommend routine screening for NAFLD in either the general population or among high-risk groups, because of uncertainties surrounding diagnostic tests and cost-effectiveness. ${ }^{16}$ However, the European Association for the Study of the Liver (EASL) does recommend screening for NAFLD with liver enzymes and/or ultrasound as part of the workup of individuals with obesity or the metabolic syndrome. ${ }^{17}$ Alternatively, some groups have recommended increased vigilance in high-risk groups, such as patients with diabetes. ${ }^{18}$ Notably, one recent study demonstrated that $11 \%$ of people with incidental hepatic steatosis on imaging are at high risk of advanced hepatic fibrosis based on the NAFLD fibrosis score and may represent a target population warranting further evaluation by a liver specialist. $^{19}$

In addition to uncertainty regarding the role of screening in NAFLD, there is a lack of public understanding about the disease that likely contributed to the low awareness noted in our cohort. Prior cross-sectional clinical studies have shown poor awareness of NAFLD as a disease entity, including among those patients at high metabolic risk. ${ }^{20,}{ }^{21}$ In one study in an outpatient endocrinology clinic, less than $20 \%$ of patients surveyed had heard of NAFLD. ${ }^{22}$ This poor understanding of NAFLD is particularly problematic given that lifestyle changes remain the most effective method for altering the disease course.

Unfortunately, poor public awareness may stem from poor disease understanding among practitioners, who may underestimate the prevalence of NAFLD and express uncertainty about its management. ${ }^{23,}{ }^{24}$ Although the majority of primary care providers in one study identified NAFLD as an important health problem, they underestimated its prevalence and only $27 \%$ reported referring NAFLD patients to a specialist in hepatology. ${ }^{23}$ Nonhepatology sub-specialists also underestimate the prevalence of NAFLD. In one survey-based study of nongastroenterology subspecialists that included endocrinologists and cardiologists, $75 \%$ of respondents estimated the prevalence of NAFLD to be $\leq 10 \%{ }^{25}$ Furthermore, practitioners may not be talking with their patients about the risks of fatty liver before it develops. In one survey of 5000 Brooklyn residents approached in a nonmedical setting, 98\% had never had a discussion with their doctor about NAFLD. ${ }^{20}$

Collectively, lack of practitioner experience combined with low patient awareness of NAFLD has potential implications for targeting "at-risk" populations for education, risk factor reduction, and appropriate referral of high-risk patients to hepatology clinics. NAFLD puts patients at higher risk for both liver-related and overall mortality. ${ }^{26}$, $27 \mathrm{NASH}$, as compared with hepatic steatosis without inflammation, is associated with more rapid progression of fibrosis. ${ }^{28}$ This is important because hepatic fibrosis is the primary predictor of liver-related mortality in NAFLD. ${ }^{29}$ Once NAFLD is identified, clinical prediction rules (e.g., NAFLD Fibrosis Score and FIB-4) and transient elastography can be used to noninvasively risk stratify patients. This allows for more targeted intervention in those at highest risk of advanced disease, which will become increasingly relevant as new therapies are approved. Counseling and risk modification are appropriate for patients at all stages of disease, and while there are no definitive data yet that such interventions will reduce liver disease progression rates, they should result in improved cardiovascular health, which is the most important cause of morbidity and mortality in NAFLD overall. Ideally, awareness of NAFLD will continue to improve among providers and the public going forward, so that patients can be risk stratified and appropriately counseled on interventions that are likely to improve metabolic and liver health.

Several limitations to this study should be noted. First, disease awareness is a result of multiple factors, including whether the diagnosis has previously been made and communicated to the patient, and whether that patient has retained the information. This analysis only reflects the end point of those processes and therefore limits our ability to interpret the underlying reasons why so few people are aware of a diagnosis of NAFLD. Second, CT has limited sensitivity for detection of hepatic steatosis compared with magnetic resonance imaging, which may have led to falsely low estimates of prevalence in this cohort. ${ }^{30}$ Third, certain data that might be helpful in characterizing an individual's severity of liver disease are not available, such as liver chemistries and noninvasive liver fibrosis scores. Additionally, the threshold for detection of hepatic steatosis used in the current study (e.g., $\leq 51 \mathrm{HU}$, which is equivalent to a liver spleen ratio $<1.0$ ) has high sensitivity but reduced specificity for diagnosis of NAFLD compared with lower liver attenuation thresholds. ${ }^{31,32}$ Thus, some CARDIA participants 
may have been misclassified as having NAFLD. However, when a lower threshold of $\leq 40 \mathrm{HU}$ (equivalent to moderate-severe hepatic steatosis) was used, the prevalence of NAFLD awareness was similar. Finally, the small number of "NAFLD aware" participants limits statistical power to assess for multiple predictors of NAFLD awareness.

In summary, our findings highlight a need for improved public and physician understanding of NAFLD, which represents an increasingly prevalent disease with significant associated morbidity and mortality. The lack of awareness demonstrated here also represents an opportunity for increased education and allocation of resources to facilitate linkage to care for patients with incidentally diagnosed NAFLD in order to prevent liver and nonliver-related adverse outcomes related to this burgeoning disease.

Acknowledgments: The authors thank the participants of the CARDIA Study for their long-term commitment and important contributions to the study.

Corresponding Author: Lisa B. VanWagner, MD, MSc, FAST, FAHA; Department of Medicine-Division of Gastroenterology \& Hepatology Northwestern University Feinberg School of Medicine, 676 N. St. Clair St., Suite 1400, Chicago, IL 60611, USA (e-mail: lvw@northwestern. edu).

Funding Information The CARDIA Study is supported by the National Heart, Lung, and Blood Institute (NHLBI) in collaboration with the University of Alabama at Birmingham (HHSN268201800005I \& HHSN268201800007I), Northwestern University (HHSN268201800003I), University of Minnesota (HHSN268201800006I), and Kaiser Foundation Research Institute (HHSN268201800004I). Dr. VanWagner is supported by the National Institutes of Health (KL2TROO1424) and the NHLBI (K23HL136891). Dr. Carr is supported by the National Institutes of Health (R01HL098445).

\section{Compliance with Ethical Standards:}

Informed consent was obtained at each follow-up examination. Institutional Review Board (IRB) approval was obtained from all study sites prior to data collection.

Conflict of Interest: The authors declare that they do not have a conflict of interest.

\section{REFERENCES}

1. Younossi ZM, Koenig AB, Abdelatif D, Fazel Y, Henry L, Wymer M. Global epidemiology of nonalcoholic fatty liver disease-Meta-analytic assessment of prevalence, incidence, and outcomes. Hepatology. 2016;64(1):73-84.

2. Younossi ZM, Stepanova M, Afendy M, Fang Y, Younossi Y, Mir H, et al. Changes in the prevalence of the most common causes of chronic liver diseases in the United States from 1988 to 2008. Clin Gastroenterol Hepatol. 2011;9(6):524-30 e1; quiz e60.

3. Chalasani N, Younossi Z, Lavine JE, Charlton M, Cusi K, Rinella M et al. The diagnosis and management of nonalcoholic fatty liver disease: Practice guidance from the American Association for the Study of Liver Diseases. Hepatology. 2018;67(1):328-57.

4. Adams LA, Lymp JF, St Sauver $\mathbf{J}$, Sanderson SO, Lindor KD, Feldstein A, et al. The natural history of nonalcoholic fatty liver disease: a population-based cohort study. Gastroenterology. 2005;129(1):113-21.
5. Argo CK, Northup PG, Al-Osaimi AM, Caldwell SH. Systematic review of risk factors for fibrosis progression in non-alcoholic steatohepatitis. J Hepatol. 2009;51(2):371-9.

6. Friedman GD, Cutter GR, Donahue RP, Hughes GH, Hulley SB, Jacobs DR, Jr., et al. CARDIA: study design, recruitment, and some characteristics of the examined subjects. J Clin Epidemiol. 1988;41(11):1105-16.

7. VanWagner LB, Ning H, Lewis CE, Shay CM, Wilkins J, Carr JJ, et al. Associations between nonalcoholic fatty liver disease and subclinical atherosclerosis in middle-aged adults: the Coronary Artery Risk Development in Young Adults Study. Atherosclerosis. 2014;235(2):599-605.

8. Piekarski J, Goldberg HI, Royal SA, Axel L, Moss AA. Difference between liver and spleen CT numbers in the normal adult: its usefulness in predicting the presence of diffuse liver disease. Radiology. 1980;137(3):727-9.

9. Kodama Y, Ng CS, Wu TT, Ayers GD, Curley SA, Abdalla EK, et al. Comparison of CT methods for determining the fat content of the liver. AJR Am J Roentgenol. 2007;188(5): 1307-12.

10. Park SH, Kim PN, Kim KW, Lee SW, Yoon SE, Park SW, et al. Macrovesicular hepatic steatosis in living liver donors: use of CT for quantitative and qualitative assessment. Radiology. 2006;239(1):105-12.

11. Warnick GR. Enzymatic methods for quantification of lipoprotein lipids. Methods Enzymol. 1986;129:101-23.

12. Friedewald WT, Levy RI, Fredrickson DS. Estimation of the concentration of low-density lipoprotein cholesterol in plasma, without use of the preparative ultracentrifuge. Clin Chem. 1972;18(6):499-502.

13. Grundy SM, Brewer HB, Jr., Cleeman JI, Smith SC, Jr., Lenfant C. Definition of metabolic syndrome: report of the National Heart, Lung, and Blood Institute/American Heart Association conference on scientific issues related to definition. Arterioscler Thromb Vasc Biol. 2004;24(2):e13-8.

14. Parker ED, Schmitz KH, Jacobs DR, Dengel DR, Schreiner PJ. Physical activity in young adults and incident hypertension over 15 years of follow-up: the CARDIA study. Am J Public Health. 2007;97(4):703-9.

15. Cleveland E, Bandy A, VanWagner LB. Diagnostic challenges of nonalcoholic fatty liver disease/nonalcoholic steatohepatitis. Clin Liver Dis (Hoboken). 2018;11(4):98-104

16. Corey KE, Klebanoff MJ, Tramontano AC, Chung RT, Hur C. Screening for Nonalcoholic Steatohepatitis in Individuals with Type 2 Diabetes: A Cost-Effectiveness Analysis. Dig Dis Sci. 2016;61(7):2108-17.

17. European Association for the Study of the L, European Association for the Study of D, European Association for the Study of O. EASL-EASDEASO Clinical Practice Guidelines for the management of non-alcoholic fatty liver disease. J Hepatol. 2016;64(6):1388-402.

18. Wong Vw, Chalasani N. Not routine screening, but vigilance for chronic liver disease in patients with type 2 diabetes. J Hepatol. 2016;64(6):12113.

19. Wright AP, Desai AP, Bajpai S, King LY, Sahani DV, Corey KE. Gaps in recognition and evaluation of incidentally identified hepatic steatosis. Dig Dis Sci. 2015;60(2):333-8.

20. Ghevariya V, Sandar N, Patel K, Ghevariya N, Shah R, Aron J, et al Knowing What's Out There: Awareness of Non-Alcoholic Fatty Liver Disease. Front Med (Lausanne). 2014;1:4.

21. Leung CM, Lai LS, Wong WH, Chan KH, Luk YW, Lai JY, et al. Nonalcoholic fatty liver disease: an expanding problem with low levels of awareness in Hong Kong. J Gastroenterol Hepatol. 2009;24(11):1786-90.

22. Wieland AC, Mettler P, McDermott MT, Crane LA, Cicutto LC, Bambha KM. Low awareness of nonalcoholic fatty liver disease among patients at high metabolic risk. J Clin Gastroenterol. 2015;49(1):e6-e10.

23. Said A, Gagovic V, Malecki K, Givens ML, Nieto FJ. Primary care practitioners survey of non-alcoholic fatty liver disease. Ann Hepatol. 2013;12(5):758-65.

24. Wieland AC, Guallick M, Truesdale A, Mettler P, Bambha KM. Identifying practice gaps to optimize medical care for patients with nonalcoholic fatty liver disease. Dig Dis Sci. 2013;58(10):2809-16.

25. Bergqvist CJ, Skoien R, Horsfall L, Clouston AD, Jonsson JR, Powell EE. Awareness and opinions of non-alcoholic fatty liver disease by hospital specialists. Intern Med J. 2013;43(3):247-53.

26. VanWagner LB, Rinella ME. Extrahepatic Manifestations of Nonalcoholic Fatty Liver Disease. Curr Hepatol Rep. 2016;15(2):75-85.

27. Ong JP, Pitts A, Younossi ZM. Increased overall mortality and liverrelated mortality in non-alcoholic fatty liver disease. J Hepatol. 2008;49(4):608-12.

28. Singh S, Allen AM, Wang Z, Prokop LJ, Murad MH, Loomba R. Fibrosis progression in nonalcoholic fatty liver vs nonalcoholic steatohepatitis: a systematic review and meta-analysis of paired-biopsy studies. Clin Gastroenterol Hepatol. 2015;13(4):643-54 e1-9; quiz e39-40. 
29. Ekstedt M, Hagstrom H, Nasr P, Fredrikson M, Stal P, Kechagias S et al. Fibrosis stage is the strongest predictor for disease-specific mortality in NAFLD after up to 33 years of follow-up. Hepatology. 2014.

30. Cho CS, Curran S, Schwartz LH, Kooby DA, Klimstra DS, Shia J, et al. Preoperative radiographic assessment of hepatic steatosis with histologic correlation. J Am Coll Surg. 2008;206(3):480-8.

31. Boyce CJ, Pickhardt PJ, Kim DH, Taylor AJ, Winter TC, Bruce RJ, et al. Hepatic steatosis (fatty liver disease) in asymptomatic adults identified by unenhanced low-dose CT. AJR Am J Roentgenol. 2010;194(3):623-8.
32. Pickhardt PJ, Park SH, Hahn L, Lee SG, Bae KT, Yu ES. Specificity of unenhanced CT for non-invasive diagnosis of hepatic steatosis: implications for the investigation of the natural history of incidental steatosis. Eur Radiol. 2012;22(5):1075-82.

Publisher's Note Springer Nature remains neutral with regard to jurisdictional claims in published maps and institutional affiliations. 